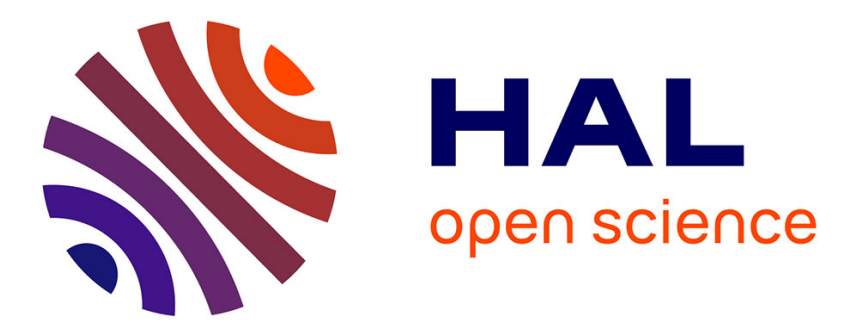

\title{
Dynamic evolution and fracture of multilayer field emitters in atom probe tomography: a new interpretation
}

Nicolas Rolland, François Vurpillot, Sébastien Duguay, Didier Blavette

\section{To cite this version:}

Nicolas Rolland, François Vurpillot, Sébastien Duguay, Didier Blavette. Dynamic evolution and fracture of multilayer field emitters in atom probe tomography: a new interpretation. European Physical Journal: Applied Physics, 2015, 72 (2), pp.21001. 10.1051/epjap/2015150233 . hal-02107622

\section{HAL Id: hal-02107622 \\ https://hal.science/hal-02107622}

Submitted on 29 Apr 2019

HAL is a multi-disciplinary open access archive for the deposit and dissemination of scientific research documents, whether they are published or not. The documents may come from teaching and research institutions in France or abroad, or from public or private research centers.
L'archive ouverte pluridisciplinaire HAL, est destinée au dépôt et à la diffusion de documents scientifiques de niveau recherche, publiés ou non, émanant des établissements d'enseignement et de recherche français ou étrangers, des laboratoires publics ou privés. 


\section{EPJ AP \\ Applied Physics}

EPJ ors

Eur. Phys. J. Appl. Phys. (2015) 72: 21001

DOI: $10.1051 /$ epjap/2015150233

\section{Dynamic evolution and fracture of multilayer field emitters in atom probe tomography: a new interpretation}

Nicolas Rolland, François Vurpillot, Sébastien Duguay, and Didier Blavette 


\title{
Dynamic evolution and fracture of multilayer field emitters in atom probe tomography: a new interpretation
}

\author{
Nicolas Rolland ${ }^{\mathrm{a}}$, François Vurpillot, Sébastien Duguay, and Didier Blavette \\ Normandie Université, Groupe de Physique des Matériaux, Université et INSA de Rouen, UMR CNRS 6634, \\ 76801 St Etienne du Rouvray, France
}

Received: 28 April 2015 / Received in final form: 3 August 2015 / Accepted: 22 September 2015 Published online: 3 November 2015 - (c) EDP Sciences 2015

\begin{abstract}
Since Atom Probe Tomography reconstruction is based on ion back projection onto the emitter surface, understanding of the evolution dynamics of the tip shape is essential to get an accurate picture of the initial sample. In this article, an analytical approach is presented to dynamically describe the morphology evolution of complex multilayer structures during field evaporation. The model is mostly founded on the common continuity hypothesis, except for the classical hemispherical description of the tip apex, which is extended to a wider class of a constant mean curvature surface of revolution, the Delaunay surfaces. The results obtained from this approach are comparable with standard numerical simulations, but the analytical character of the model gives more insight into the principles driving the emitter morphology. In particular, a complete picture of curvature evolution during the transition from one layer to another is provided. Additionally, a field evaporation threshold for tip fracture in a bilayer sample is highlighted.
\end{abstract}

\section{Introduction}

Atom probe tomography has proven to be a unique characterization tool for a wide range of applications, and especially in the field of microelectronics [1-3]. The outstanding depth resolution, revealing atomic planes, makes it an ideal candidate for the analysis of thin layers. However, it is known that many artefacts are present in the reconstruction of such a structure, due to inhomogeneous evaporation fields, which in turn result in complex emitter morphology [4]. Indeed, ion back projection onto a hemispherical surface is at the core of every reconstruction protocol [5-10].

While great efforts have been made in the field of numerical simulation to understand the surface shape evolution of such a sample [4,11-17], there is presently no theoretical framework accounting for this phenomenon. In a previous study, we demonstrated that a few simple ingredients were necessary to reproduce common artefacts in the reconstruction [18]. Here, we present a model in the same vein based on an original mathematical definition of the emitter surface shape. Indeed, we extent the classical hemispherical representation to a larger class of constant mean curvature surfaces of revolution, the Delaunay surfaces [19]. Those surfaces, which are usually used to model the surface of a liquid film [20], are perfectly suited to the description of field evaporated surfaces if one assumes

\footnotetext{
a e-mail: nicolas.rolland1@univ-rouen.fr
}

the common relationship between an electric field and the mean radius of curvature $(F \propto 1 / R)$.

The modeling is applied to a complex multilayer structure in order to compare the computed shape of the emitter with results obtained from state-of-the-art numerical simulation. Both methods give quite similar results, but the analytical character of the present approach makes it possible to highlight precisely the mechanisms governing the shape evolution dynamics. In particular, we show that it is relatively incomplete to state that during the transition from one layer to another the radius of curvature of the phase with higher evaporation field decreases, while it increases for the phase with the lower evaporation field. Eventually, we highlight a field ratio threshold between layers, above which we might expect tip fracture.

\section{Modeling}

\subsection{Hypothesis}

The present modeling deals with the evolution of the field emitter shape during atom probe analysis, when the sample is made up of several phases stacked in layers perpendicular to the tip axis, in the optimum configuration to analyze layers. In contrast, layers parallel to the tip axis lead to major trajectory overlaps due to local magnification effects and more biased composition data. Similar to the approaches used in APT reconstruction 
algorithms, the atomic details of the surface are ignored in the present approach and the surface has been represented at a mesoscopic scale by a smooth continuous mathematical function. Basically, the initial sample prior to field evaporation is then depicted as a hemispherical cap of radius $R_{0}$ seated on a truncated cone with shank angle $\alpha$. As a specific adaptation for our model, we now introduce $N$ interfaces delimiting $N+1$ layers stacked in the emitter tip (Fig. 1a). Each interface $i$ is labelled with its depth $z_{i}$ and its field ratio $f_{i}$. This field ratio is define as follows: considering an interface separating a layer of material B seated on a layer of material A, the interface is assigned with the field ratio $f=F_{B}^{E} / F_{A}^{E}$ where $F_{B}^{E}$ and $F_{A}^{E}$ are the zero-barrier evaporation fields of material $\mathrm{B}$ and $\mathrm{A}$ respectively (Fig. 1b). Note that this field ratio can be tuned to take into account specific operating conditions, as shown in reference [18]. Now, the aim of this study is to describe the emitter shape evolution when material is removed by field evaporation.

To this end, we introduce the basic relationship between the emitter mean radius of curvature $R$ and the surface field $F[21]$ :

$$
F=\frac{V}{k_{f} R},
$$

where $V$ is the electric potential applied to the tip and $k_{f}$ is a geometrical factor known as the field factor (note, this formula is only valid for positive $R$ ). In the following, we assume that this formula can be applied locally over any surface point, with a constant factor $k_{f}$. From this consideration, one can infer several rules governing the emitter shape evolution:

1. There is tangential continuity all over the emitter surface. Otherwise $R$ would be locally null and $F$ infinite: the surface residence time of such a point (i.e., atom) is null.

2. Each phase of the sample develops a constant mean curvature surface (e.g., the hemispherical cap when the sample is homogeneous). Indeed, the progressive removal of atoms with high evaporation probability results in a surface with a constant evaporation probability, and thus a constant electric field.

3. For the same reason, the mean radii of curvature of two different phases $\mathrm{A}$ and $\mathrm{B}$ compensates the evaporation field differences as follows:

$$
\frac{R_{A}}{R_{B}}=\frac{F_{B}^{E}}{F_{A}^{E}} .
$$

Actually, these simple rules have been known for many decades: in particular, statements (1) and (2) form the cornerstone of the original reconstruction algorithm developed at the early stage of APT [5].

However, no reconstruction method has been proposed to account for sample heterogeneities according to equation (2). In a recent article, we have already tried to establish the foundations of such a method for a bilayered tip [18]. Here, we present a more general and reliable model based on an original mathematical representation of the emitter surface.

\subsection{Delaunay surfaces}

According to rule (2), and pointing out that the studied geometry exhibits rotational symmetry, the Delaunay surfaces appear as a natural representation of the emitter shape. Indeed, those surfaces obtained by rotating the roulettes of the conics are the only surfaces of revolution with constant mean curvature [19]. They can be divided into six distinct groups, according to the nature of the original conics: the plane, the catenoid, the cylinder, the sphere, the unduloid and the nodoid. The first two groups are not of interest for the present study since their mean curvature is zero. In addition, the cylinder and the sphere can be obtained as particular cases of the onduloid class; thus we only have to deal with the onduloid and nodoid surfaces. The latter are obtained by rotating the roulettes of the ellipse and the hyperbola respectively, and are entirely determined by the half-axes $a$ and $b$ of the corresponding conics as depicted in Figure 2.

From the well-defined procedure represented in Figure 2a to compute an unduloid, one can infer the construction of the cylinder and the sphere as particular cases of the unduloid. Indeed, if $a=b$ the ellipse turns into a circle, and the resulting curve gives a cylinder. If $b=0$, the ellipse turns into a segment and the resulting curve gives a series of spheres. Also, note that the nodoid presents a self-intersection, rendering the inner part of the surface unusable.

Figure 3 is a diagram outlining the principles of the present model. At each stage of evaporation, layers subjected to field evaporation are assigned with a specific Delaunay surface. This translates into a set of $N+1$ trios of parameters $\left(a_{i}, b_{i}, \varepsilon_{i}\right)$ representing the associated conics. Indeed, on the one hand $\varepsilon_{i}=1$ for an unduloid, obtained from the rotation of an elliptic roulette and $\varepsilon_{i}=-1$ for a nodoid, obtained from the rotation of a hyperbolic roulette. On the other hand, $a_{i}$ and $b_{i}$ are the half-axes of the conics.

We now make use of rules (1) and (3) to set those parameters. At this stage of the discussion, it is important to recall the expression of the local mean radius of curvature $R$ for a surface of revolution [19]:

$$
\frac{1}{R}=\frac{1}{2}\left(\frac{1}{R_{C}}+\frac{1}{N}\right)
$$

where $R_{C}$ is the local radius of curvature of the generatrix (the curve rotated around the revolution axis) and $N$ is the distance between the generatrix and the revolution axis, in the direction of the local surface normal (Fig. 4).

Fortunately, it is quite straightforward to express those values for a Delaunay surface. For a surface point at a distance $r$ from the revolution axis, it writes as:

$$
\begin{aligned}
N & =\varepsilon_{i} \frac{2 a_{i} r^{2}}{b_{i}^{2}+\varepsilon_{i} r^{2}} \\
R_{C} & =-\varepsilon_{i} \frac{2 a_{i} r^{2}}{b_{i}^{2}-\varepsilon_{i} r^{2}} \\
R & =2 a_{i} .
\end{aligned}
$$


N. Rolland et al.: Dynamic evolution and fracture of multilayer field emitters in atom probe tomography

(a)

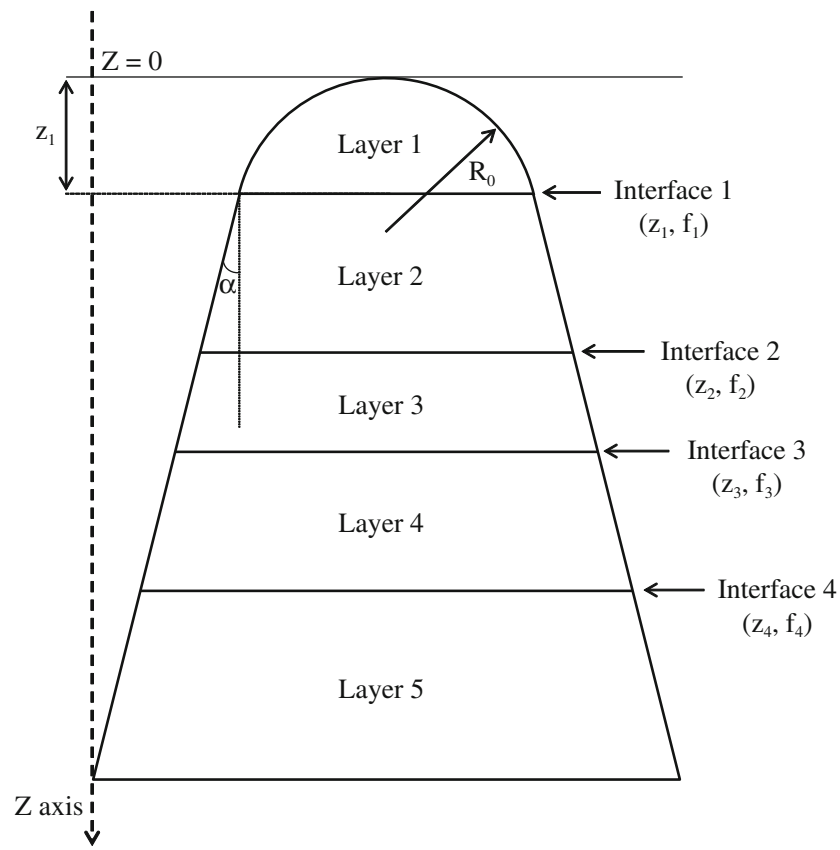

(b)

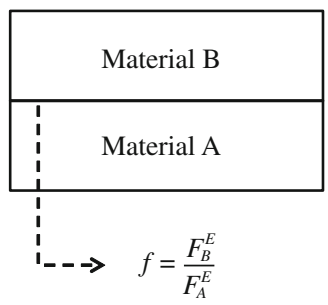

Fig. 1. (a) Classically, the initial tip shape is modelled by a hemispherical cap with radius $R_{0}$ seated on a truncated cone with shank angle $\alpha$. The sample is made up of several layers stacked in layers perpendicular to the tip axis. They are separated by interfaces, that are assigned with their depth $z$ and their field ratio $f$, (b) a layer of material B seated on a layer of material A. The field ratio is defined as the ratio of the evaporation fields: $f=F_{B}^{E} / F_{A}^{E}$.

(a)

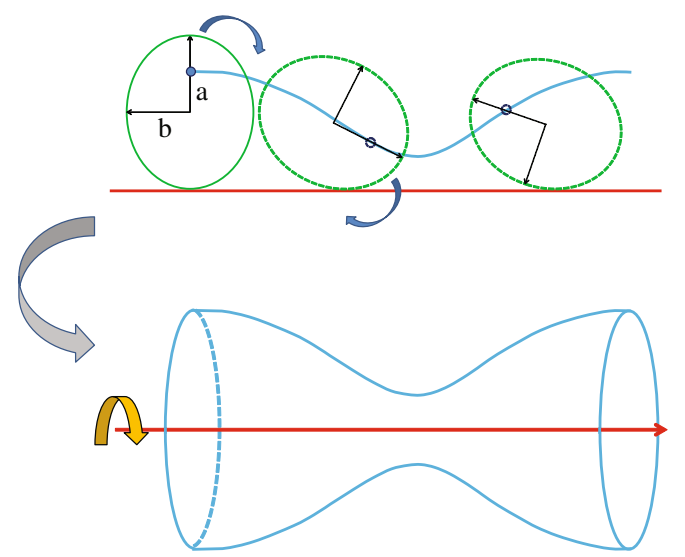

(b)

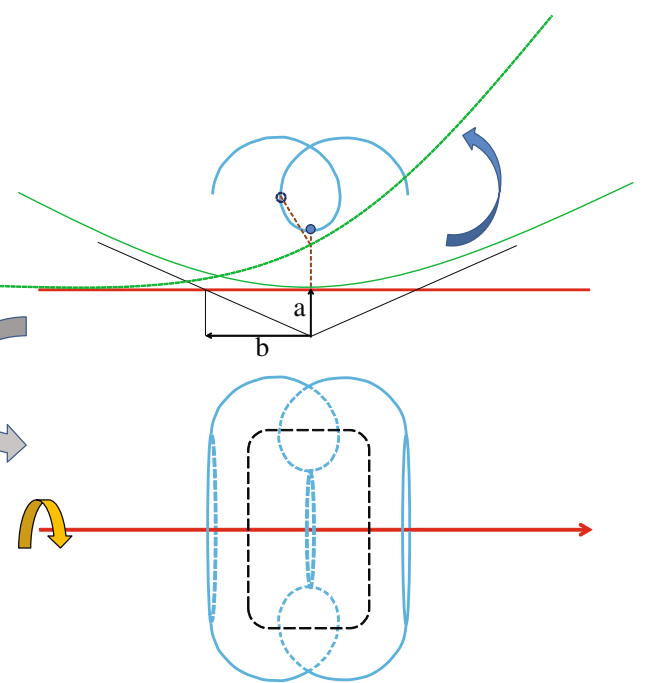

Fig. 2. (a) The roulette of the ellipse is found by tracing out a focus of an ellipse, rolling without friction on an axis. Rotating this roulette around the same axis gives the constant mean curvature surface called unduloid, (b) the roulette of the hyperbola is found by tracing out a focus of a hyperbola rolling without friction on an axis. Green lines are the hyperbola. The second hyperbola (dashed line) is obtained by rolling the first hyperbola toward the left side. The dashed brown lines point toward the focus of each hyperbola, which generate the roulette. The asymptotes of the first hyperbola are shown as a black line to evidence the semi-axes $a$ and $b$ as indicated by black arrows. Rotating this roulette around the red axis gives the constant mean curvature surface called a nodoid. In practice, the inner part of the nodoid in the dotted frame is unused since the normal is oriented toward the revolution axis. 


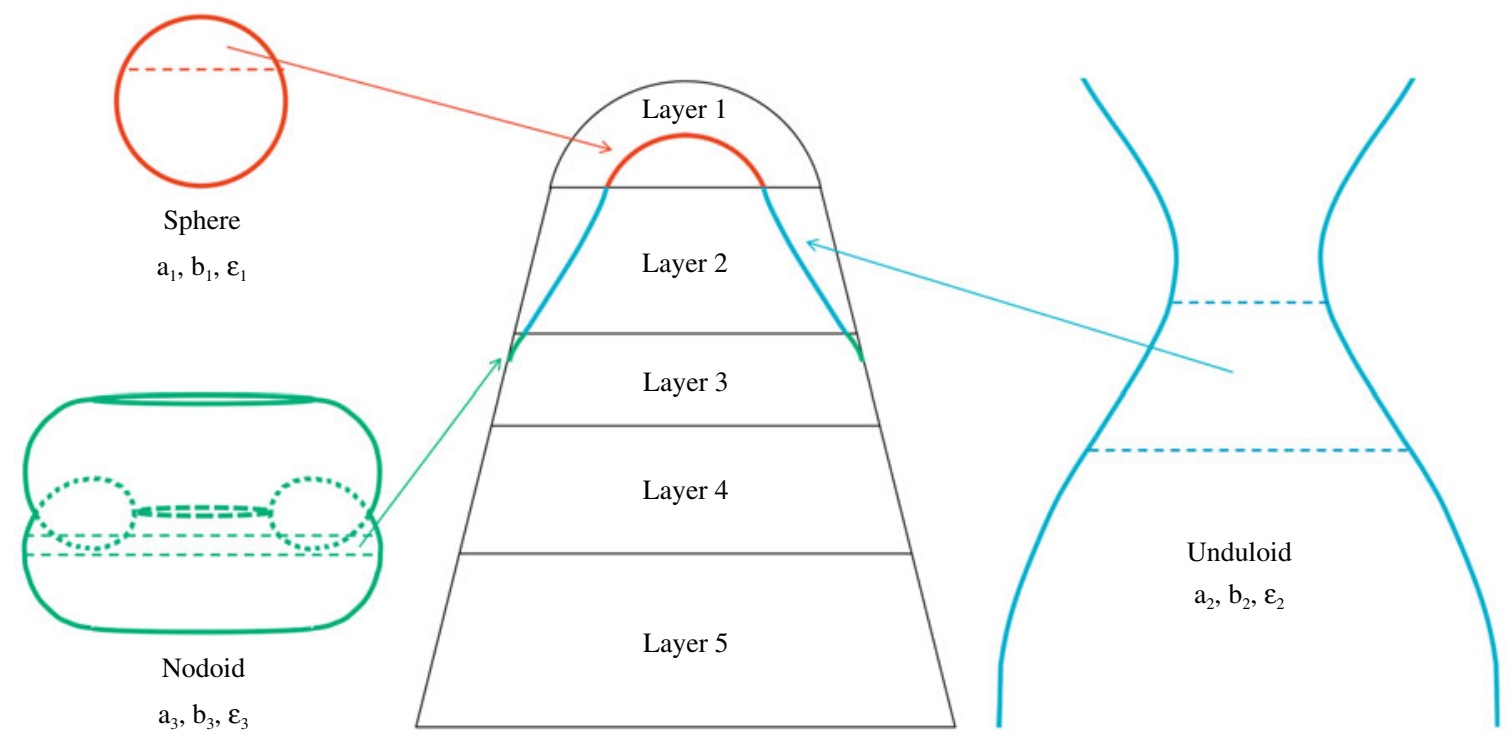

Fig. 3. The field evaporated surface is modelled by a set of Delaunay surfaces. Each layer, i.e., each different material, is modelled by a specific surface, parametrized by the trio of parameters $\left(a_{i}, b_{i}, \varepsilon_{i}\right)$.

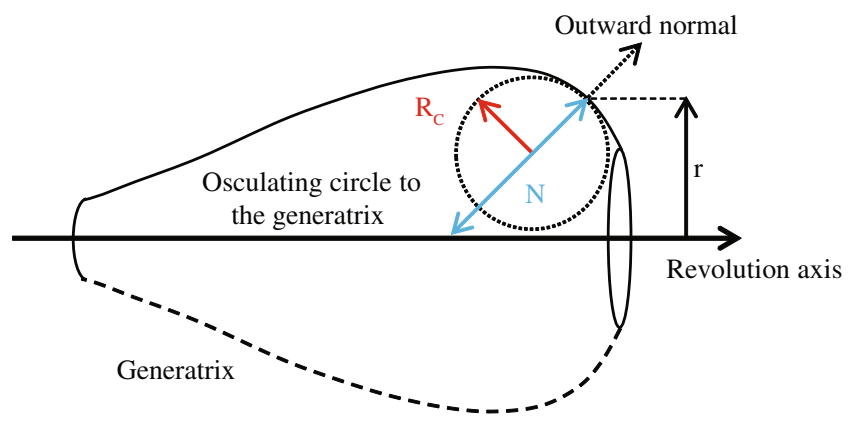

Fig. 4. The mean curvature of a surface of revolution equates to the average of the curvature $1 / R_{C}$ of the generatrix and of the inverse normal length $1 / N$.

Now consider layers $i$ and $i+1$, separated by the interface $i$. Equation (2) together with the geometrical formulae of equation (4) immediately yields:

$$
a_{i+1}=a_{i} \frac{R_{i+1}}{R_{i}}=\frac{a_{i}}{f_{i}}
$$

And since there is tangential continuity over the tip surface (rule (1)), there is equality across the normal, $N$, on both sides of the interface at a distance $r_{c}$ from the revolution axis. Thus one can infer:

$$
\varepsilon_{i+1} \frac{2 a_{i+1} r_{c}^{2}}{b_{i+1}^{2}+\varepsilon_{i+1} r_{c}^{2}}=\varepsilon_{i} \frac{2 a_{i} r_{c}^{2}}{b_{i}^{2}+\varepsilon_{i} r_{c}^{2}} .
$$

Injecting equation (5) and rearranging leads to:

$$
b_{i+1}^{2}=\varepsilon_{i+1}\left(b_{i}^{2}+r_{C}^{2}\left(\frac{1}{f_{i}}-1\right)\right) .
$$

The role of this equation is two-fold. First, it defines unambiguously the sign of $\varepsilon_{i+1}$, and thus the nature of the $i+1$ th Delaunay surface. Also, it gives an expression for $b_{i+1}$ in relation to $b_{i}$.

\subsection{Dynamic shape evolution}

In the previous section, we introduced a set of expressions establishing a link between the parameters of layer $i+1$ and $i$. We shall now explain how one can compute the emitter shape evolution during evaporation. In practice, the parametrization of the roulettes is rather complicated, and this makes it impossible to obtain an analytical formula describing this dynamic evolution. Thus, a numerical resolution of the problem was adopted.

The progressive evaporation of the emitter is described as a step by step process. Each of those step correspond to the evaporation of a material of thickness $d z_{e}$ at the tip apex. Figure 5 depicts the principles of the algorithm used to solve the problem, with only two layers for the sake of simplicity. The initial emitter shape is the black line. The first step of the algorithm is to find the emitter shape after removal of a material of thickness $d z_{e}$. This shape is denoted by (2) on Figure 5 (red and blue thick lines). It is worth mentioning that the top layer of the tip (red thick line) is necessarily a truncated sphere. Indeed, this is the only Delaunay surface that crosses the revolution axis and one can infer $\varepsilon_{\text {top }}=1$ and $b_{\text {top }}=0$. In addition, it was demonstrated in the previous section that parameters of layer $i+1\left(a_{i+1}, b_{i+1}, \varepsilon_{i+1}\right)$ can be deduced from the knowledge of parameters of layer $i\left(a_{i}\right.$, $\left.b_{i}, \varepsilon_{i}\right)$. Therefore, the value of $a_{\mathrm{top}}$, the half radius of curvature of the hemispherical cap (red thick line), sets the shape of all the underlying layers (blue thick line). In particular, one can see that the solution we are looking for (surface (2)) ensures the tangential continuity of the surface at the base of the field evaporated area. So, we end 


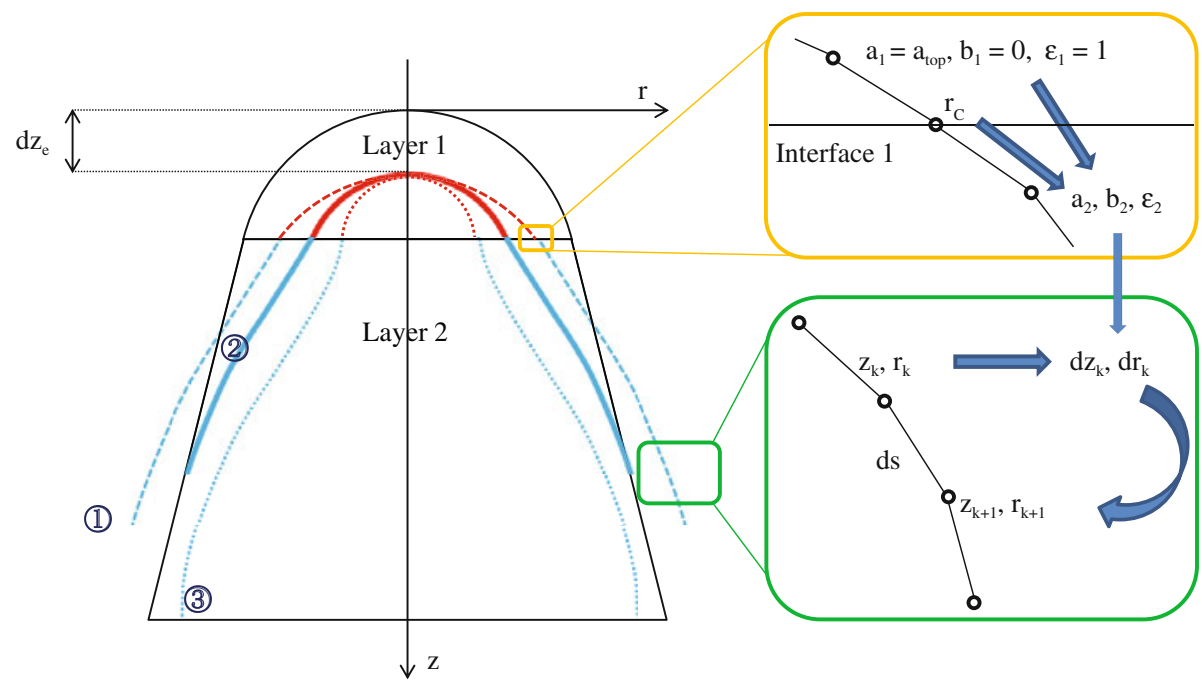

Fig. 5. Numerical resolution of the geometric problem. The green frame illustrates the use of equation (8) to compute the surface of one of the layers. The orange frame illustrates the use of equations (5) and (7) to compute how the surface parameters change across the interface. Surface (2) corresponds to the solution for $a_{\text {top }}$, resulting in a tangential continuity at the base of the field evaporated area. Surface (1) corresponds to the crossing with the previous surface: $a_{\mathrm{top}, t}$ has to be decreased. Surface (2) corresponds to a surface which never crosses the initial surface: $a_{\mathrm{top}, t}$ has to be increased.

up with the problem of finding value $a_{\text {top }}$ corresponding to the removal of a material of thickness $d z_{e}$ on the tip axis, such that there is tangential continuity at the base of the field evaporated area.

To solve this geometric problem, several values of $a_{\text {top }}$ are tried and the tangential continuity condition is checked for each of them. In practice, the continuity is checked by computing a discrete representation of the emitter shape. With this aim, we introduce a small $d s$ (corresponding to the curvilinear abscissa step) and the shape is computed step by step. Indeed, the following formulae hold for the roulettes of the conics:

$$
\begin{array}{r}
d z=d s \frac{b_{i}^{2}+\varepsilon_{i} r^{2}}{2 a_{i} r} \\
d r=d z \sqrt{\frac{4 a_{i}^{2} r^{2}}{\left(r^{2}+\varepsilon_{i} b_{i}^{2}\right)^{2}}-1},
\end{array}
$$

where $d r$ and $d z$ are respectively the radial and in-depth infinitesimal displacements along the surface (see axes $r$ and $z$ in Fig. 5). Thus, from a point $\left(r_{k}, z_{k}\right)$ on the surface, the point $\left(r_{k+1}, z_{k+1}\right)$ is easily obtained as $r_{k+1}=r_{k}+d r$ and $z_{k+1}=z_{k}+d z$ (green frame in Fig. 5). The whole surface computation proceeds as follows. We shall denote $a_{\mathrm{top}, t}$ a test value for $a_{\mathrm{top}}$, and $d s$ the curvilinear abscissa step. Considerer that $a_{\mathrm{top}, t}$ corresponds to surface (1) in Figure 5 . The first point $\left(r_{0}, z_{0}\right)$ of the surface is $\left(0, d z_{e}\right)$. The first layer shape (red dashed line) is computed step by step from (8) $\left(a_{1}=a_{\mathrm{top}, t}, b_{1}=0\right.$ and $\left.\varepsilon_{1}=1\right)$. When interface 1 is reached at $r_{c},(5)$ and $(7)$ are used to compute the parameters $\left(a_{2}, b_{2}, \varepsilon_{2}\right)$ (orange frame in Fig. 5 ). Again, we make use of (8) with those new parameters to compute the shape of the second layer (blue dashed line). In this particular case, one can see that the computed shape crosses the initial surface, and thus the continuity condition is not fulfilled. Indeed, $a_{\mathrm{top}, t}$ is too large compared to the solution we are looking for (surface (2)).

Based on this remark, the test values $a_{\text {top }}$ are chosen following a binary search algorithm. This algorithm consists of searching the solution $a_{\text {top }}$ in an interval $\left[a_{\text {top, min }}, a_{\text {top, max }}\right]$. Obviously, the interval has to be large enough to ensure that the solution is in between the boundaries. The first test value for $a_{\text {top }}$ is chosen as the mean value of the interval boundaries: $a_{\mathrm{top}, t}=\left(a_{\mathrm{top}, \min }+\right.$ $\left.a_{\text {top,max }}\right) / 2$. The corresponding shape is then computed as explained in the previous paragraph. If the surface crosses the initial surface (surface (1) in Fig. 5), $a_{\mathrm{top}, t}$ is too large compared to the solution (surface (2)). Therefore, the search interval becomes $\left[a_{\mathrm{top}, \mathrm{min}}, a_{\mathrm{top}, t}\right]$. On the contrary, if the surface never crosses the initial surface (surface (3)), $a_{\mathrm{top}, t}$ is too small compared to the solution and the search interval becomes $\left[a_{\mathrm{top}, t}, a_{\mathrm{top}, \mathrm{max}}\right]$. A second test value for $a_{\text {top }}$ is then chosen as the mean value of the new interval boundaries and the corresponding shape is computed. The interval is subsequently modified according to the rules mentioned above. The process is repeated until the interval size is below a given threshold $\Delta a$ (which turns to be the accuracy of the solution). $a_{\text {top }}$ is then the mean value of the last interval boundaries.

Eventually, the shape of the emitter is known after removal of a material of thickness $d z_{e}$ on the tip axis. This surface becomes the "initial surface", and a new surface is computed following the same process after removal of a total material of thickness $2 d z_{e}$. Step by step, this algorithm provides a dynamic picture of the emitter morphology all along the field evaporation process.

This semi-analytical approach provides a fast way to compute the dynamic evolution of the emitter shape, which is of special interest in order to understand 
artefacts due to local magnification: indeed, the mean radius of curvature of each phase is known all through the evaporation process. In the following section, we will compare our semi-analytical approach with finite-differences numerical simulations, with a special focus on the emitter curvature evolution during field evaporation of multilayer structures. For this purpose, one needs to compare the emitter morphology at the same "moment" of evaporation. From the present modelling, it would appear natural to consider the depth of evaporation on the tip axis as a scale of the evaporation progress. However, it is much more common in the literature to evaluate this progress from the number of evaporated atoms, or similarly, from the evaporated volume. Note, it is also in this form that atom probe experimental raw datasets are collected. Consequently, to each step of the present algorithm, corresponding to the removal of a material of thickness $d z_{e}$, we associate an evaporated volume on the emitter surface. This computation is done once the dynamic evolution of the emitter has entirely been computed. As mentioned previously, our modelling provides a discrete representation of the surface at successive instants of evaporation (corresponding to successive removal of thickness $d z_{e}$ ). Figure 6 depicts the output of such a computation. The points contained in orange cells delimit the initial emitter shape (note that only half of the emitter has to be considered due to symmetry). All the others are points computed from the application of our algorithm. Consider the points contained in green cells. Those points delimit the emitter surface at the same step of the algorithm, after removal of a total thickness $4 d z_{e}$ on the tip axis. In order to associate an evaporated volume to this surface, a Voronoï partition of the whole set of surface points obtained from the modeling is computed: it results in the cell partition that one can see in Figure 6. Each point is then assigned with a virtual atomic volume $V_{\text {at }}=2 \pi r A_{\text {cell }}$, where $r$ is the distance from the point to the revolution axis and $A_{\text {cell }}$ is the area of the corresponding Voronoï cell. Eventually, the evaporated volume corresponding to the green surface is obtained by summing the atomic volumes of the points being part of it. Thus, we are able to discuss the emitter morphology evolution as a function of the evaporated volume of matter, as in Figure 7 for instance.

\section{Basic properties of the modeling}

In this section, we demonstrate that the simple ingredients introduced previously are sufficient to reproduce the basic features of field evaporation usually highlighted by finitedifference numerical simulations. Additionally, we discuss common assumptions related to the curvature evolution during the transition from one layer to the other. We thus identify a field ratio threshold above which the tip fracture is expected.

\subsection{A complete description of curvature evolution}

Numerous studies have been conducted to understand the shape evolution of multilayer structured emitters by the

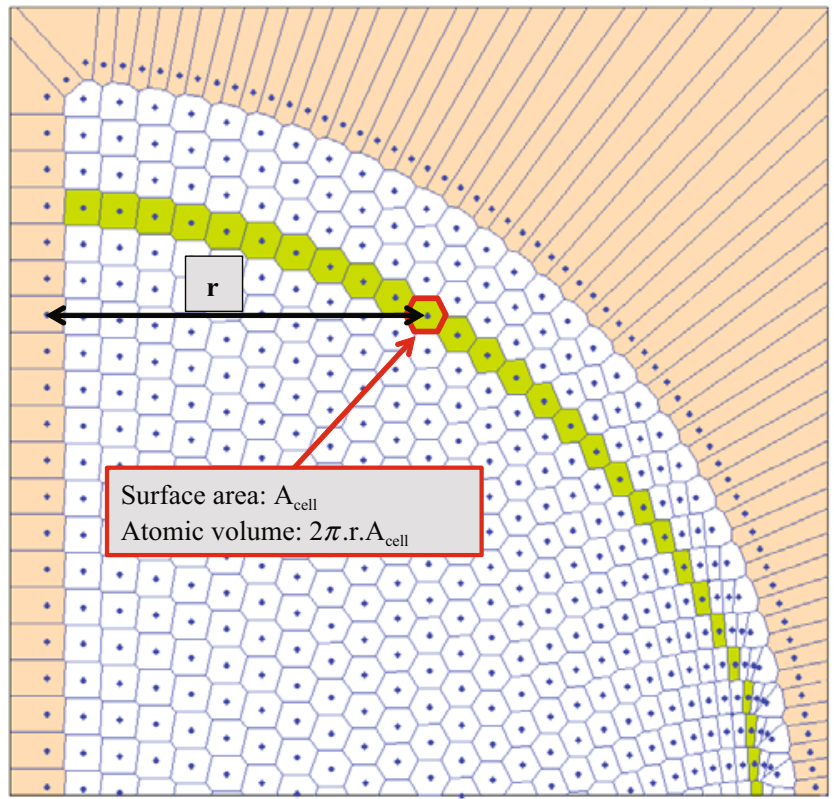

Fig. 6. Voronoï partition of the whole set of points obtained from the modeling. Cells filled in orange delimit the initial emitter shape. Cells filled in green correspond to one specific surface obtained from the modelling. The evaporated volume corresponding to this surface is obtained by summing all the individual atomic volumes of green cells.

mean of atomic scale numerical simulation. They consistently found that during the transition from one layer to the other, the radius of curvature of the phase with higher evaporation field decreases, while it increases for the phase with the lower evaporation field $[12,17]$. In order to clarify this point, we have modeled the evaporation of an emitter structure similar to that presented in reference [17] since the modification of the apex curvature was rigorously tracked. This structure is made up of three layers with different evaporation fields stacked in a cylindrical shaft with a radius of $25 \mathrm{~nm}$. The stack consists of a high field top layer (blue), a low field intermediate layer (red), and a high field bottom layer (green), see Figure 7 . The field ratio of the first interface is set to $f=1.5$ while it is set to $f=0.25$ for the second interface. The evolution of the emitter shape is presented together with the mean curvature evolution of each layer (Fig. 7). Note here that we plotted the mean curvature, which is simply the inverse of the mean radius of curvature.

The computed shapes agree well with the results presented in reference [17]. In particular, the unduloid surface modeling the intermediate red layer is ideally suited to represent the formation of a cone like shape (2nd picture of Fig. 7a), a characteristic feature of the transition from a high evaporation field layer (top blue layer) to a low evaporation field layer (intermediate red layer). Also, the nodoid surface modeling the bottom green layer makes it possible to depict the retention of higher evaporation field atoms at the emitter apex (3rd picture of Fig. 7a). With regards to the mean curvature evolution 
N. Rolland et al.: Dynamic evolution and fracture of multilayer field emitters in atom probe tomography

(a)

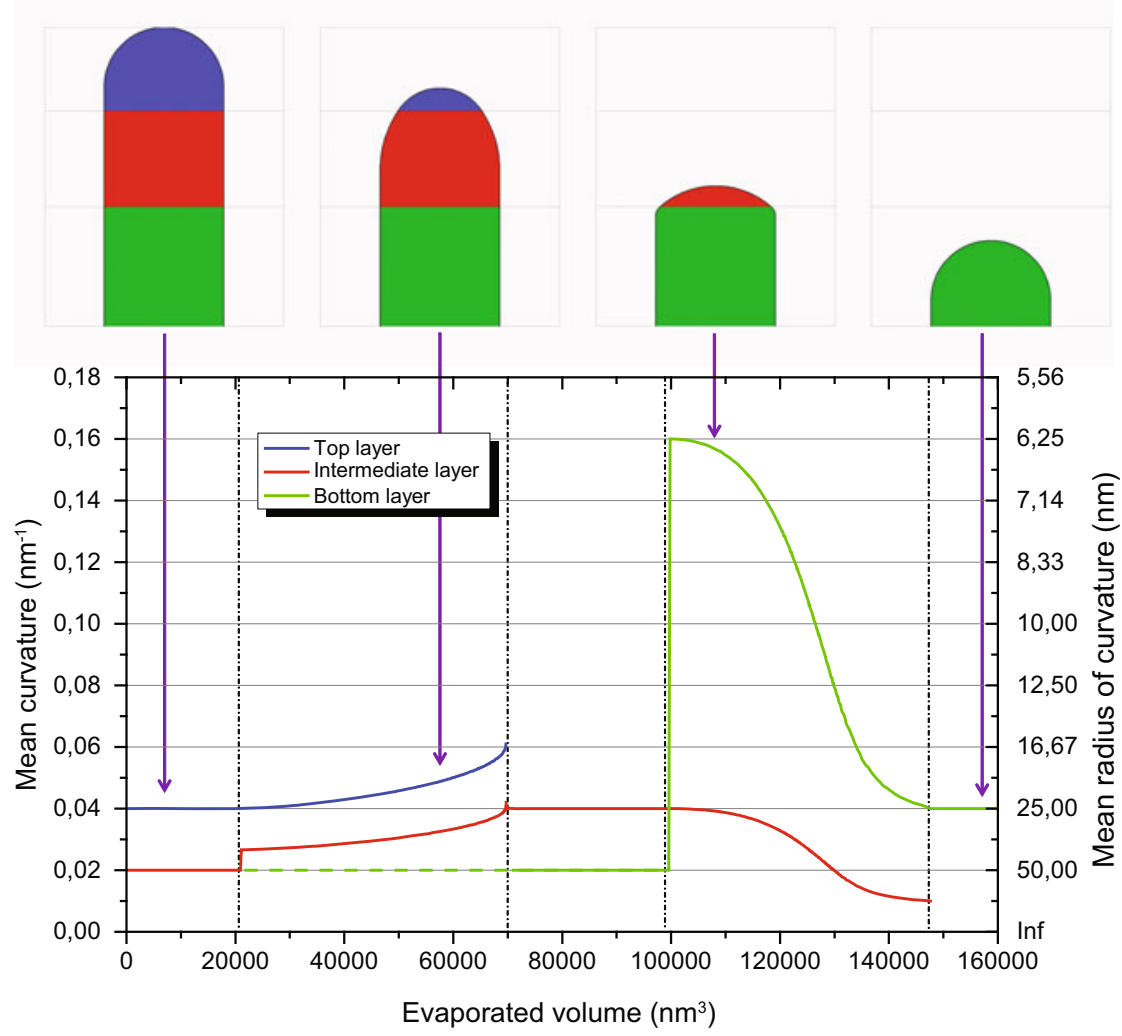

Fig. 7. (a) Emitter shape during field evaporation. The top blue layer is a high field material, the intermediate red layer is a low field material and the bottom green layer is again a high field material. The field ratio of the first interface (between blue layer and red layer) is set to $f=1.5$ while it is set to $f=0.25$ for the second interface (between red layer and green layer), (b) mean curvature evolution for each layer of the structure. The color code corresponds to (a).

during evaporation, there is again good qualitative agreement with numerical simulation studies. However, we are not entirely happy with the finding stating that the radius of curvature of the phase with higher evaporation field decreases (blue and green curves), while it increases for the phase with the lower evaporation field (red curve). Indeed, it is clear from the plot in Figure 1b that during the transition from the top blue layer (high field) to the intermediate red layer (low field), mean curvatures of both phases are increasing (mean radii of curvature of both phases are respectively decreasing). Note that this evolution is also quite unambiguous in the plots reported in reference [17]. The evolution is more subtle for the transition from the intermediate red layer (low field) to the bottom green layer (high field). When the first atoms of the bottom layer are evaporated, there is a sharp rise of the mean curvature of the corresponding surface (green curve around $100000 \mathrm{~nm}^{3}$, Fig. 7b), followed by a slower decrease. Indeed, one can see that once the high evaporation field surface curvature has reached its maximum value, both layer curvatures decrease and this is what drives the emitter morphology. Note that the sharp rise of the curvature from the value of the cylindrical shaft curvature $\left(0.02 \mathrm{~nm}^{-1}\right)$ to a higher value also happens during the first transition from the top layer to the intermediate layer (red curve around $20000 \mathrm{~nm}^{3}$, Fig. 7b), showing that this is not a characteristic feature of the low on high transition. The curvature evolution during the transition from one layer to another is summarized as follows:

- when the bottom layer started to evaporate, there was a sharp evolution of its curvature toward a value of $\gamma / f$, where $\gamma$ is the top layer mean curvature and $f$ is the field ratio of the interface, as a direct consequence of equation (2). This evolution can be an increase or a decrease depending on the field ratio and the initial bottom layer curvature (preset by the cylindrical or conical shaft);

- then, either both layer mean curvatures increase (high on low case) or decrease (low on high case) together to ensure that $\gamma_{\text {top }} / \gamma_{\text {bottom }}=f$ throughout the evaporation.

\subsection{A field ratio threshold for tip fracture}

We now consider a structure made up of a layer, B, seated on a layer, A, and separated by an interface with field ratio $f$. Although it has been known for a long time that large values of $f$ can lead to tip fracture, we shall now highlight a quantitative value for the stability limit. In a previous study, we demonstrated that below a critical 


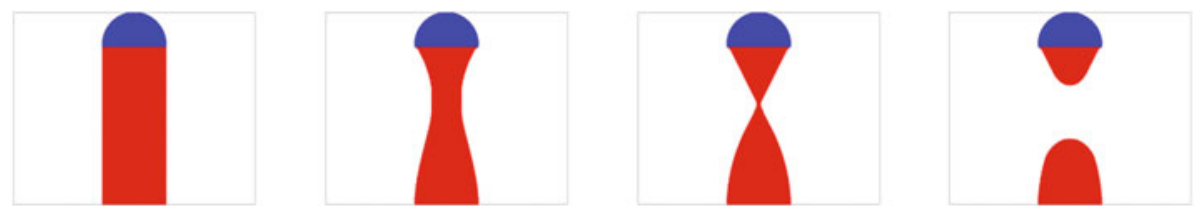

Fig. 8. Evaporation sequence of a bilayer structure. The field ratio at the interface is 2.5 . The low evaporation field red layer is evaporating while the blue layer is not, eventually resulting in a fracture of the tip.

value of field ratio $f=2$, the bottom layer will develop a negative meridian radius of curvature $R_{C, A}$ at the interface [18]. This is directly seen by injecting equation (3) in equation (2):

$$
\frac{\frac{1}{R_{C, B}}+\frac{1}{N_{B}}}{\frac{1}{R_{C, A}}+\frac{1}{N_{A}}}=f .
$$

Noting that there is tangential continuity of the surface at the interface and that the top layer is forced to be modelled by a hemispherical cap as explained in the modeling section, one can infer that $N_{A}=N_{B}=R_{C, B}$ and thus, rearranging equation (9) leads to:

$$
R_{C, A}=\frac{R_{C, B}}{\frac{2}{f}-1} .
$$

Note this expression is only valid at the interface. Nevertheless, since $R_{C, B}$ is the positive meridian radius of the hemispherical cap, one can note that $f>2$ implies that $R_{C, A}$ is negative. This in turn, forces the bottom layer to be modeled by an unduloid surface since this is the only Delaunay surface with negative meridian radius of curvature. Let us now assume that the emitter structure is confined in a cylindrical shaft of mean radius of curvature $R_{\text {cyl }}$. Thus, the mean radius of curvature of the hemispherical cap is $R_{\text {cyl }} / 2$ before the evaporation of the bottom layer has started. From equation (1), one can see that the electric field above the hemispherical surface is $2 F_{\text {cyl }}$, where $F_{\text {cyl }}$ is the electric field above the cylindrical surface. But the field ratio is strictly superior to 2, and therefore the bottom layer starts to evaporate before the top layer. The former develops an unduloid shape confined in the cylindrical shaft. Unfortunately, this evolution decreases the mean radius of curvature of the bottom layer surface. Indeed, the cylinder is a particular case of unduloid, and more precisely this is the unduloid that maximizes the mean radius of curvature of the surface. Thus, the field surface above the bottom layer is even higher, and eventually the whole bottom layer evaporates before the top layer starts to evaporate: it might results in a tip fracture. In order to confirm this unexpected behavior highlighted by the present modeling, we simulated the evaporation of a bilayer structure with a field ratio $f=2.5$, using the numerical simulation code presented in reference [14]. As predicted, the bottom low evaporation layer is evaporating while the tip layer is not (Fig. 8), eventually resulting in tip fracture. Also, an unduloid shape is clearly seen, validating the use of Delaunay surfaces to model the field emitter shape.

\section{Conclusion}

A semi-analytical model has been presented to describe the field evaporation of complex multilayer structures. The cornerstone of the approach is the use of Delaunay surfaces to model the surface of the emitter. Indeed, those constant mean curvature surfaces of revolution are naturally suited to model constant field evaporation probability surface. Assuming the basic relationship between electric field and curvature $(F \propto 1 / R)$, the model exhibits comparable features to those obtained from conventional numerical simulations. We modeled the dynamic shape evolution of a complex multilayer structure and demonstrated that the transition from one layer to another is a two steps process. First, there is a sharp evolution of the bottom layer curvature toward a value compensating the field evaporation difference between both layers. Then, both layer mean curvatures increase or decrease together to keep their ratio constant throughout the evaporation. Additionally, we highlighted a field ratio threshold $f=2$, corresponding to a top layer field evaporation amounting to twice the value of the bottom layer, above which we might expect a tip fracture. Note that the present approach is easily generalizable to any emitter structure with revolution symmetry. Further work will focus on the integration of an ion projection law in the modeling to get a complete picture of multilayer structure evaporation. We hope this analytical approach will eventually lead to improvements of the reconstruction method.

The author acknowledges the support of the French Agence Nationale de la Recherche (ANR) under the reference ANR12-NANO-0001.

\section{References}

1. A.K. Kambham, J. Mody, M. Gilbert, S. Koelling, W. Vandervorst, Ultramicroscopy 111, 535 (2011)

2. A. Grenier, S. Duguay, J.P. Barnes, R. Serra, G. Haberfehlner, D. Cooper, F. Bertin, S. Barraud, G. Audoit, L. Arnoldi, E. Cadel, A. Chabli, F. Vurpillot, Ultramicroscopy 136, 185 (2014)

3. A. Grenier, S. Duguay, J.P. Barnes, R. Serra, N. Rolland, G. Audoit, P. Morin, P. Gouraud, D. Cooper, D. Blavette, F. Vurpillot, Appl. Phys. Lett. 106, 213102 (2015)

4. F. Vurpillot, D. Larson, A. Cerezo, Surf. Interface Anal. 36, 552 (2004) 
5. P. Bas, A. Bostel, B. Deconihout, D. Blavette, Appl. Surf. Sci. 87-88, 298 (1995)

6. B. Gault, D. Haley, F. de Geuser, M.P. Moody, E.A. Marquis, D.J. Larson, B.P. Geiser, Ultramicroscopy 111, 448 (2011)

7. B. Gault, S.T. Loi, V.J. Araullo-Peters, L.T. Stephenson, M.P. Moody, S.L. Shrestha, R.K.W. Marceau, L. Yao, J.M. Cairney, S.P. Ringer, Ultramicroscopy 111, 1619 (2011)

8. S.K. Suram, K. Rajan, Ultramicroscopy 132, 136 (2013)

9. B. Geiser, D. Larson, E. Oltman, S. Gerstl, D. Reinhard, T. Kelly, T. Prosa, Microsc. Microanal. 15, 292 (2009)

10. F. Vurpillot, B. Gault, B.P. Geiser, D.J. Larson, Ultramicroscopy 132, 19 (2013)

11. E.A. Marquis, B.P. Geiser, T.J. Prosa, D.J. Larson, J. Microsc. 241, 225 (2011)

12. D.J. Larson, B.P. Geiser, T.J. Prosa, S.S.A. Gerstl, D.A. Reinhard, T.F. Kelly, J. Microsc. 243, 15 (2011)

13. D.J. Larson, B.P. Geiser, T.J. Prosa, T.F. Kelly, Microsc. Microanal. 18, 953 (2012)
14. F. Vurpillot, A. Gaillard, G. Da Costa, B. Deconihout, Ultramicroscopy 132, 152 (2013)

15. D. Haley, M.P. Moody, G.D.W. Smith, Microsc. Microanal. 19, 1709 (2013)

16. C. Oberdorfer, S.M. Eich, G. Schmitz, Ultramicroscopy 128, 55 (2013)

17. C. Oberdorfer, S.M. Eich, M. Lütkemeyer, G. Schmitz, Ultramicroscopy (2015), DOI: 10.1016/j.ultramic. 2015.02.008

18. N. Rolland, D.J. Larson, B.P. Geiser, S. Duguay, F. Vurpillot, D. Blavette, Ultramicroscopy (2015), DOI: $10.1016 /$ j.ultramic.2015.03.010

19. C. Delaunay, J. Mathématiques Pures Appliquées 6, 309 (1841)

20. J.A.F. Plateau, Statique Expérimentale et Théorique Des Liquides Soumis Aux Seules Forces Moléculaires (Gauthier-Villars, Paris, 1873)

21. R. Gomer, Field Emission and Field Ionization (Harvard University Press, Cambridge, Massachusetts, 1961) 